\title{
Influence of the Changed USP Specifications on Disintegration Test Performance
}

\author{
Katja Schmid ${ }^{1}$ and Raimar Löbenberg ${ }^{2 *}$ \\ 'Department of Pharmacy-Pharmaceutical Technology and Biopharmaceutics, \\ Ludwig-Maximilians-University Munich, 81377 Munich, Germany \\ ${ }^{2}$ Faculty of Pharmacy and Pharmaceutical Sciences, University of Alberta, \\ Edmonton, AB, T6G 2N8, Canada
}

\begin{abstract}
The aim of this study was to investigate if the changes made in the specifications of the disintegration procedure impact the performance of the disintegration test described in USP chapters $<701>$ and $<2040>$. Different tablets and capsules were produced, and their disintegration times were determined. The following disintegration time parameters were analyzed: volume of the immersion fluid, type of apparatus (Apparatus A for method <701 >; Apparatus B for method $<2040>$ ), and attachment of a wire cloth to the basket assembly. By adjusting the compaction force and lubricant level, the disintegration time of the tablets was standardized to $15 \mathrm{~min}$. The disintegration time change was statistically significant when varying the volume of the immersion fluid. The type of apparatus and the attachment of a wire cloth resulted in no significant difference in the disintegration time of capsules. The USP requirements for immersion medium volume should be strictly followed to obtain correct and reproducible test results. The disintegration test is a suitable performance test for certain pharmaceutical and dietary dosage forms.
\end{abstract}

\section{INTRODUCTION}

D isintegration was the first performance test for solid oral dosage forms required by the Swiss Pharmacopoeia back in 1935 (1). The rationale for disintegration testing is given by the fact that most oral dosage forms need to fragment into powder particles before drug particles are released and dissolved. Yet, disintegration is no direct measure for drug dissolution and cannot be used as a universal in vivo bioavailability predictor (2). The disintegration test simply determines if the solid oral dosage form under investigation disintegrates in a given time frame. However, for BCS class I drugs, disintegration rather than dissolution was lately discussed as a possible performance test (3). The dissolution of class I and class III drugs is not limited within the physiological $\mathrm{pH}$ range (4), and here, disintegration can be seen as the critical step for bioavailability (5). Using a Quality by Design (QbD) approach, a correlation between drug particle size and dissolution rate might be established, and subsequently, dissolution testing might be substituted by disintegration testing (3). Due to the establishment of $\mathrm{QbD}$, disintegration might again become an important performance test.

According to the USP, disintegration of a pharmaceutical or dietary dosage form is a performance test that is intended to ensure the batch-to-batch consistency of a product. USP 32 describes three different apparatus that can be used to perform a disintegration test. Chapter

*Corresponding author.

Dissolution Technologies | FEBRUARY 2010
$<701>$ describes Apparatus A consisting of a basket assembly with six observation cylinders. Chapter $<2040>$ describes Apparatus B, which uses observation cylinders with a larger diameter. Both apparatus are well-established, and their designs have hardly changed over the past decade. Donauer et al. (5) reviewed the current specification changes for the disintegration apparatus and test conditions. USP 32 general chapter $<2040>$ lists a third method for the disintegration of soft-shell capsules. In the "rupture test," the opening of soft-shell capsules is the performance test criterion.

Scientific data describing the disintegration test is limited (6-9). In recent years, USP changed the procedure of the disintegration test and harmonized the chapter $<701>$ with the European and Japanese Pharmacopoeia (10). The test conditions prior to USP 28 only specified that the bottom wire mesh of the observation cylinder should have a distance of at least $25 \mathrm{~mm}$ from the bottom of the beaker on the downward stroke and at least $25 \mathrm{~mm}$ from the fluid level on the upward stroke. Now, USP states the same downward stroke specification but specifies that the basket assembly should not be totally submerged and the distance of the wire mesh should be at least $15 \mathrm{~mm}$ from the fluid level on the upward stroke (Figure 1). In the previous specifications, a wire mesh was applied to keep the dosage forms from floating out of the observation cylinders on the downward stroke, while the new specification makes the upper wire cloth obsolete. However, it is still part of the USP text (11). To the best of our knowledge, the impact of these changes on the performance of the disintegration test has not been investigated. 


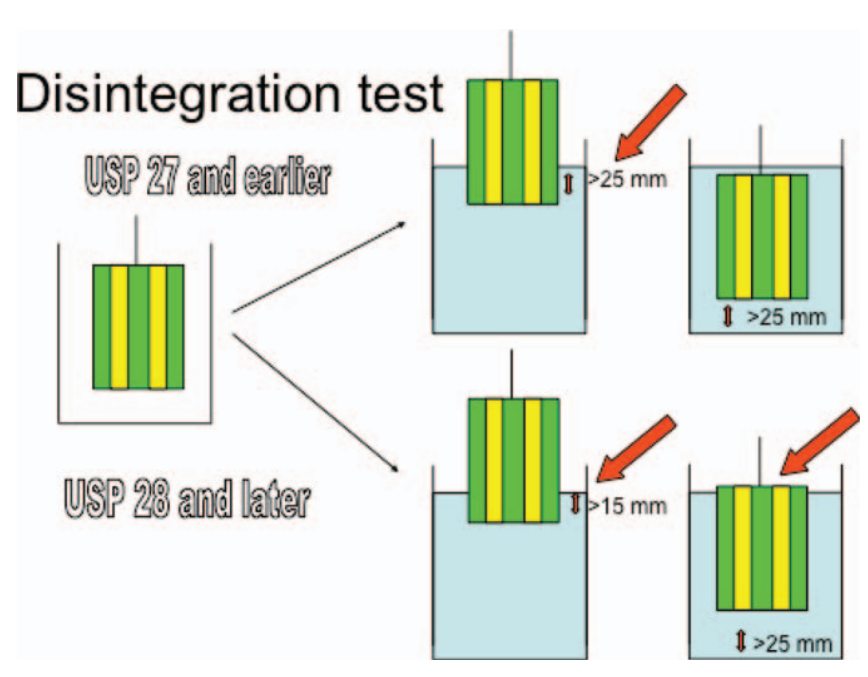

Figure 1. Changes in dissolution test conditions: USP 27 and earlier allowed submersion of the basket assembly; USP 28 and later prohibit complete basket immersion.

The aim of this study was to determine if the changes made to the disintegration test procedure impact the disintegration times and, therefore, the performance of the disintegration test. Test dosage forms were manufactured, and formulation and manufacturing parameters were investigated to identify suitable test units. Then, the disintegration behavior of selected test units was assessed under different fluid-level conditions and apparatus.

\section{MATERIALS AND METHODS \\ Excipients}

The following materials were used: GranuLac 200 (lactose monohydrate, Meggle, Germany), Methocel E5 PR LV (hydroxypropyl methylcellulose, Dow Chemicals, USA), magnesium stearate (Street Chemical \& Co, Canada), talc and Aerosil (Degussa AG, Germany), ethyl alcohol (99\%), and empty hard gelatin capsules \# 1 (Wiler-PCCA, Canada).

\section{Tablet Compositions and Manufacturing Processes}

The following basic tablet composition (Table 1) was used for wet granulation, and the granulate was then varied in the amount of magnesium stearate added to it (0\%, $1 \%$, and $2 \%)$. The dry excipients GranuLac and Methocel were thoroughly blended in a mortar using a pestle. While mixing continued, ethyl alcohol was added until the powder was sufficiently wet. The mass was then screened through a 1400-mesh sieve and dried for $30 \mathrm{~min}$ at $40^{\circ} \mathrm{C}$ in a shelf dryer. Sieve analysis was performed, and granules having an appropriate particle size were mixed with talc, magnesium stearate, and Aerosil before being compressed into tablets.
Table 1. Tablet Composition

\begin{tabular}{lc}
\hline Excipient & Amount \\
\hline Granulate: & \\
GranuLac & $95 \%$ \\
Methocel & $5 \%$ \\
Ethyl alcohol 50\% & q.s. \\
\hline Tablet: & \\
Granulate & $93.9 \%$ \\
Talc & $5 \%$ \\
Magnesium stearate & 1,2, or $3 \%$ \\
Aerosil & $0.1 \%$ \\
\hline
\end{tabular}

\section{Tablet Compaction}

Granulate (300 $\pm 1 \mathrm{mg}$ ) was weighed separately for each tablet and then compressed with a hydraulic laboratory press (Carver, USA) using 11.8-mm diameter, flat-faced punches. The compaction force was 1, 2, or 3 metric tons for $30 \mathrm{sec}$.

\section{Capsule Filling}

The empty hard-gelatin capsules were filled with GranuLac using a manual capsule-filling machine (Aponorm, Germany).

\section{Tensile Strength}

The hardness of the tablets was determined using a tablet hardness tester (C-DHT 200, Campbell Electronics, India), and the tensile strength was calculated by $T=2 P / H D \pi$, where $T$ is the tensile strength $\left(\mathrm{N} / \mathrm{m}^{2}\right), H$ the thickness of the tablet $(\mathrm{mm}), D$ the diameter of the tablet $(\mathrm{mm})$, and $P$ the applied fraction force $(\mathrm{N})$.

\section{Disintegration Tests}

The disintegration tester (ED-2L, Betatek, Canada) consisted of two stations, each operated with USP Apparatus A (chapter $<701>$ ) or Apparatus B (chapter $<2040>$ ). All tablet disintegration tests were performed using Apparatus A or B without disks. In addition, capsules were analyzed in Apparatus A and B with and without a wire cloth attached to the top of the basket assembly. Disintegration times were determined using the built-in digital clock of the disintegration tester. Purified water at $37 \pm 2{ }^{\circ} \mathrm{C}$ was used as immersion fluid. The current USP specifications make it necessary to determine the required amount of immersion fluid for each beaker individually because the size of the beakers might differ (USP 32 allows a beaker diameter of 97-115 mm). The amount of immersion fluid was varied between 710 and $750 \mathrm{~mL}$ for the two beakers used in this study. Furthermore, the volume of the immersion fluid was varied to simulate the previous USP specification of at least $25-\mathrm{mm}$ distance between lower wire cloth and fluid level at the upward stroke. In these tests, the larger beaker was used with $870 \mathrm{~mL}$ instead of $750 \mathrm{~mL}$ of immersion medium. In 


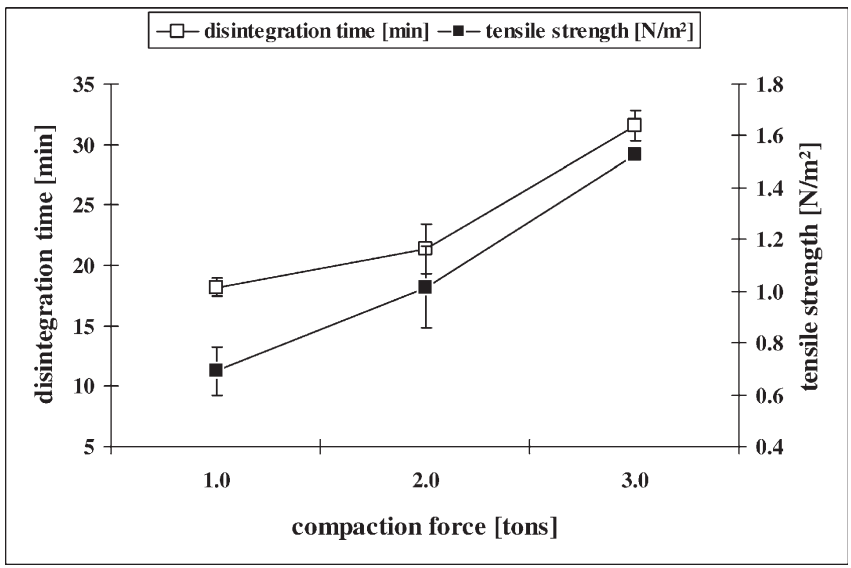

Figure 2. Disintegration time ( $\square$ ) and tensile strength ( $\square$ ) of various test tablets in relation to the compaction force used for their production $(n \geq 3)$.

addition, the impact of insufficient fluid levels was investigated, and an immersion fluid volume of $630 \mathrm{~mL}$ instead of $750 \mathrm{~mL}$ was used. When using the high fluid level of $870 \mathrm{~mL}$, a wire cloth was put on the top of the basket assembly to prevent the test units from floating out of the tubes.

\section{Disintegration Using a Paddle Apparatus}

The disintegration time of the test tablets was determined using a dissolution tester (VK 7000, VanKel, USA). The tablet disintegration was tested in $900 \mathrm{~mL}$ distilled water at $37 \pm 0.5^{\circ} \mathrm{C}$ at $50 \mathrm{rpm}$ using USP Apparatus 2 (paddle).

\section{Statistical Analysis}

An unpaired, one-tailed $t$-test was used to test the significance of difference. A $P$-value of 0.05 was defined to be statistically significant.

\section{RESULTS AND DISCUSSION}

The aim of this study was to determine if the changes made in the specifications of the disintegration procedure impact the performance of the disintegration test. Hence, test tablets were developed which were suitable to distinguish between the test conditions of the previous and the new specifications.

\section{Development of Test Tablets}

The compaction forces to press the test tablets were varied to produce tablets with an ideal disintegration behavior. The resulting tablets were analyzed for disintegration time and tensile strength. As shown in Figure 2, increasing the compaction force from 1.0 to 3.0 tons led to a prolonged disintegration time of approximately $32 \mathrm{~min}$ and an increased tensile strength of $1.5 \mathrm{~N} / \mathrm{m}^{2}$. Tablets that were compressed with 1 metric ton showed a disintegration time closest to the desired

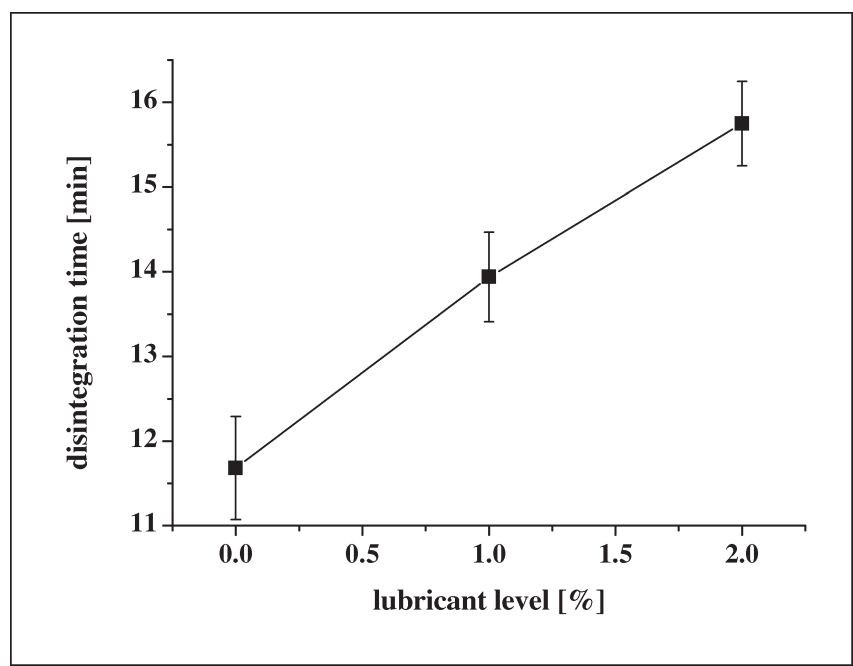

Figure 3. Disintegration time of various test tablets containing different amounts of magnesium stearate as lubricant $(n \geq 6)$.

time range of about $15 \mathrm{~min}$. The relationship between compaction force, tablet hardness, and disintegration time has been described previously (12-14), and our results are in line with these reports. The rather long disintegration time of 15 min was chosen deliberately, because a faster disintegration might not provide enough sensitivity to observe differences in the disintegration times within a reasonable sample size (15).

To establish a reliable test tablet, it is important to know the impact of changes in excipient composition on disintegration time $(16,17)$. Tablets containing $1 \%$ magnesium stearate disintegrated within the targeted time period of approximately $15 \mathrm{~min}$ and showed sufficient lubrication (Figure 3). Magnesium stearate can cause a delay in tablet disintegration (18). With an increasing level of magnesium stearate, the disintegration time was prolonged, which presumably was due to the hydrophobic nature of the lubricant coating the powder surfaces (19). Tablets containing $1 \%$ magnesium stearate that were produced by applying 1 metric ton compression force were used for all subsequent tests.

\section{Disintegration Using a Paddle Apparatus}

The disintegration time of the tablets in USP dissolution Apparatus 2 (paddle) was considerably longer than the time observed in a disintegration tester $(65.9 \pm 0.8 \mathrm{~min}$ instead of about $15 \mathrm{~min}$ ). The significant prolongation of the disintegration time was due to the absence of any destructive mechanical up-and-down forces in the paddle apparatus. Use of a separate apparatus under different hydrodynamic conditions confirmed that the tablets were sensitive to changes in hydrodynamic conditions (20) and might be sensitive to changes in the disintegration procedure (22). 


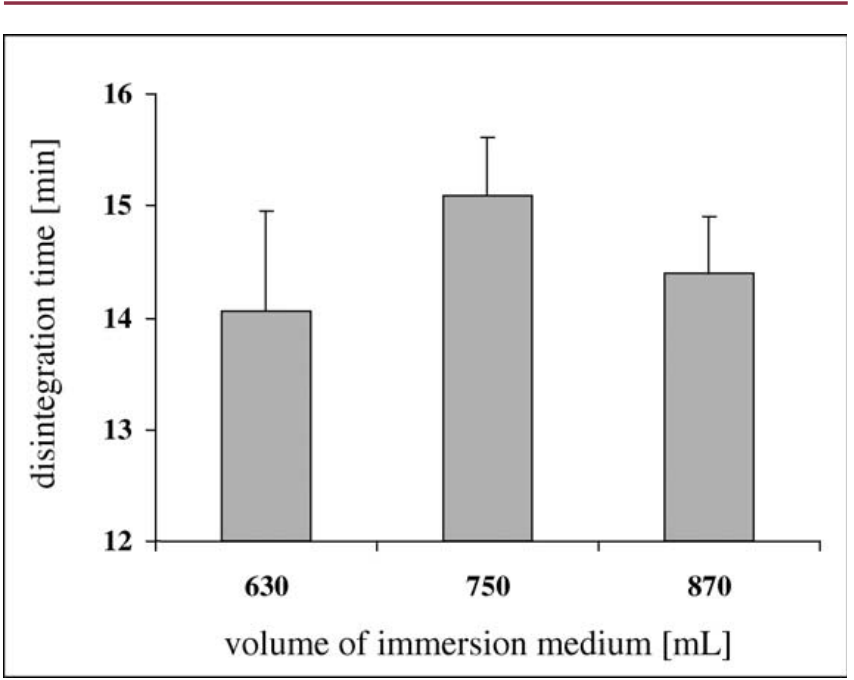

Figure 4. Disintegration time vs volume of immersion fluid ( $n \geq 10)$.

\section{Influence of the Volume of Immersion Fluid on the Disintegration Time}

The influence of the immersion fluid volume on the disintegration time was investigated in two experiments. First, the exact amount of water was determined for each beaker according to the current USP specifications. Although both beaker sizes were within the specified dimensions, one beaker needed $750 \mathrm{~mL}$, while the other needed $710 \mathrm{~mL}$. All tests performed according to the USP specifications met the expectations. To simulate slightly out-of-specification test conditions, the larger beaker was filled with the volume needed for the smaller beaker and vice versa. Although this resulted in slightly inappropriate water levels, the disintegration time of the tablets was not significantly influenced $(n=24, P>0.05)$. Apparently, conditions that are slightly out of specification do not affect the disintegration times and the test performance.

In a second approach, the amount of immersion fluid was further varied (Figure 4). If the water level was $15 \mathrm{~mm}$ higher than the old USP specification $(870 \mathrm{~mL}$ instead of $750 \mathrm{~mL}$ ) or lower than required by the current USP specification $(630 \mathrm{~mL}$ instead of $750 \mathrm{~mL})$, the disintegration time decreased. These differences in disintegration times were statistically significant $(P<0.05)$. A possible explanation might be that the tablets are moved out of the immersion medium during the upward stroke of the basket if the water level is too low. Thereby, the wire cloth on the bottom of the basket can impose a higher force on the tablets and cause a faster disintegration. On the other hand, a fluid level far above the specified dimension seems to change the hydrodynamic conditions as well. As a consequence, the performance of the disintegration test might have changed with the implementation of the new test conditions. Pharmaceutical or dietary solid dosage forms that were developed under the old conditions might show different disintegration times when tested under the new conditions.
Table 2. Capsule Disintegration Times $(n \geq 12)$

\begin{tabular}{lcc}
\hline & \multicolumn{2}{c}{$\begin{array}{c}\text { Disintegration Time } \\
\text { (min) }\end{array}$} \\
\hline Condition & Apparatus A & Apparatus B \\
\hline With wire cloth & $4.1 \pm 0.5$ & $4.1 \pm 0.6$ \\
\hline Without wire cloth & $4.4 \pm 0.5$ & $4.1 \pm 0.7$ \\
\hline
\end{tabular}

The influence of the amount of immersion fluid on the disintegration time of tablets was previously evaluated by Kamba et al. (15). In that experiment, auxiliary disks were used to accelerate the disintegration of the tablets. Presumably because of these disks, the authors of this study could not observe a significant effect of the change of water volume on the disintegration time. The use of disks shortens the disintegration time of most products (21). The shorter test time might decrease the discriminating power of the disintegration test and might contribute to the statistical insignificance. Therefore, we performed our tests without the use of auxiliary disks.

\section{Influence of the Apparatus on the Disintegration Time of Capsules}

In addition to tablets, capsules were tested using both disintegration Apparatus A and B. According to USP 32 chapter $\langle 2040\rangle$, units $18 \mathrm{~mm}$ and larger have to be tested in Apparatus B. Chapter $<701>$ does not specify any size limitations for Apparatus A. However, the ICH guidelines state that the harmonized disintegration test is only valid for units under $18 \mathrm{~mm}$ (10). Capsules with a size of $19 \mathrm{~mm}$ were chosen to be tested in both apparatus. These experiments enabled a direct comparison of the influence of the basket assembly and the wire cloth on the disintegration time. However, as shown in Table 2, the disintegration times in Apparatus A and B were not significantly different $(P>0.05)$. Thus, either apparatus appears adequate for the testing of capsules. In a second step, the wire cloth, which ensures the total immersion of the capsules at the beginning of an experiment, was attached to the top of the beakers. The contact of the capsules with the wire cloth on the downward stroke of the beaker might impact the disintegration times. However, the difference in the disintegration times between using the wire cloth or not was not statistically significant $(P>0.05)$.

\section{CONCLUSIONS}

Standardization of pharmaceutical test apparatus and procedures is one of the most important tasks to ensure reliable test results, which prevent the rejection of product batches that might actually meet the specified quality criteria. The disintegration test is a well-established performance test. However, there are very limited scientific data available that describe the disintegration test and the 
parameters that influence disintegration time. Our study showed that the changes in fluid level specifications impact the performance of the test. The USP requirements for volume of immersion medium should be strictly followed to obtain correct and reproducible test results. When the new test conditions were applied, the wire cloth described in chapter $<701>$ for capsules seemed to be irrelevant and had no impact on the test results.

\section{REFERENCES}

1. History of Dissolution Testing, 2000. Dissolution Solutions Network Web site. http://www. dissolutionsolutions.net/information/history.html (accessed Jan 14, 2010).

2. Wagner, J.G.;Wilkinson, P. K.; Sedman, A. J.; Stoll, R. G. Failure of USP tablet disintegration test to predict performance in man. J. Pharm. Sci. 1973, 62 (5), 859-860.

3. Loebenberg, R. Dissolution Testing and Quality by Design (QbD), 2008. Scitopics Web site. http://www. scitopics.com/Dissolution_Testing_and_Quality_by_ Design_QbD.html (accessed Jan 14, 2010).

4. Loebenberg, R.; Amidon, G. L. Modern bioavailability, bioequivalence and biopharmaceutics classification system. New scientific approaches to international regulatory standards. Eur.J.Pharm. Biopharm. 2000, 50 (1), 3-12.

5. Donauer, N.; Loebenberg, R. A mini review of scientific and pharmacopeial requirements for the disintegration test. Int. J. Pharm. 2007, 345 (1-2), 2-8.

6. Kwan, K. C.; Swart, F. O.; Mattocks, A. M. Factors affecting tablet disintegration.J.Am. Pharm. Assoc. 1957, 46 (4), 236-239.

7. Kaplan, L. L. Modified USP Tablet Disintegration Apparatus.J. Pharm. Sci. 1964, 53 (4), 447-449.

8. Wagner, J.G. Biopharmaceutics 14. Disintegration of Dosage Forms in Vitro and in Vivo Part IV. Drug Intel. Clin. Pharm. 1969, 11 (3), 324-330.

9. Lowenthal, W. Disintegration of tablets. J. Pharm. Sci. 1972,61 (11), 695-711.

10. International Conference on Harmonisation. Q4B Annex 5: Evaluation and Recommendation of Pharmacopoeial Texts for Use in the ICH Regions on Disintegration Test General Chapter, 2009. http://www. ich.org/cache/compo/363-272-1.html\#Annex5 (accessed Jan 14, 2010).
11. United States Pharmacopeia and National Formulary USP 32-NF 27. The United States Pharmacopeial Convention, Inc.: Rockville, MD, 2009.

12. Dedhiya, M. G.; Woodruff, C.W.; Menard, F. A.; Rhodes, C. T. Relationship Between Compression Profile and Physical Properties of Lithium Carbonate Formulation. Drug Dev. Ind. Pharm. 1988, 14 (1), 53-61.

13. Kitazawa, S.; Johno, I.; Ito, Y. Effects of hardness on the disintegration time and the dissolution rate of uncoated caffeine tablets. J. Pharm. Pharmacol. 1975, 27 (10), 765-770.

14. Miyamoto, Y.; Ryu, A.; Sugawara, S.; Miyajima, M.; Matsui, M.; Takayama, K.; Nagai, T. Optimization of the granulation process for designing tablets. Chem. Pharm. Bull. 1998, 46 (9), 1432-1437.

15. Kamba, M.; Seta, Y.;Takeda, N.; Hamaura, T.; Kusai, A.; Nakane, H.; Nishimura, K. Measurement of agitation force in dissolution test and mechanical destructive force in disintegration test. Int. J. Pharm. 2003, 250 (1), 99-109.

16. Czarnecki,W.; Baj, T. Disintegration rate and release of aminophenazone from tablets. Acta Pol. Pharm. 1997, 54 (4), 287-291.

17. Durga, P.S. Effect of a dispersant on the dissolution of acetaminophen from capsule formulations. Indian Drugs 1998, 35 (7), 434-437.

18. Kato, H.; Kimura, K.; Izumi, S.; Nakamichi, K.; Danjo, K.; Sunada, $\mathrm{H}$. The effect of magnesium stearate particle size on tablet properties and tableting characteristics of granules prepared with standard formulation. J. Drug Deliv. Sci. Tec. 2005, 15 (6), 475-480.

19. Shah; A.C.; Mlodozeniec, A. R. Mechanism of surface lubrication: influence of duration of lubricantexcipient mixing on processing characteristics of powders and properties of compressed tablets.J. Pharm. Sci. 1977, 66 (10), 1377-1378.

20. Morihara, M.; Aoyagi, N.; Kaniwa, N.; Katori, N.; Kojim, S. Hydrodynamic Flows Around Tablets in Different Pharmacopoeial Dissolution Tests. Drug Dev. Ind. Pharm. 2002, 28 (6), 655-662.

21. Loebenberg, R.; Steinke, W. Investigation of vitamin and mineral tablets and capsules on the Canadian market. J. Pharm. Pharm. Sci. 2006, 9 (1), 40-49.

22. Carstensen, J.T.; Mehta, A.; Zoglio M. A. Correlation between dissolution and disintegration in dissolution apparatuses. J. Pharm. Sci. 1983, 72 (2), 28-29. 